\title{
OFF-POCKET ACTIVITY CLIFFS, A PUZZLING FACET OF MOLECULAR RECOGNITION
}

Tigran M. Abramyan†, Yi An†, and Dmitri Kireev ${ }^{\star}$

tCenter for Integrative Chemical Biology and Drug Discovery, Division of Chemical Biology and Medicinal Chemistry, Eshelman School of Pharmacy, University of North Carolina at Chapel Hill, Chapel Hill, North Carolina, 27599-7363

*e-mail: dmitri.kireev@unc.edu

\section{Supporting information}

\section{SUPPLEMENTARY FIGURES}

The distributions in Figures S1-5 were plotted using the data obtained in four independent seeds of molecular dynamics simulations for each molecular system. The data for ligand-protein complexes involving active ligands are plotted with solid green lines, the data for systems with inactive ligands, with dashed red lines. Y-axis represents normalized counts.
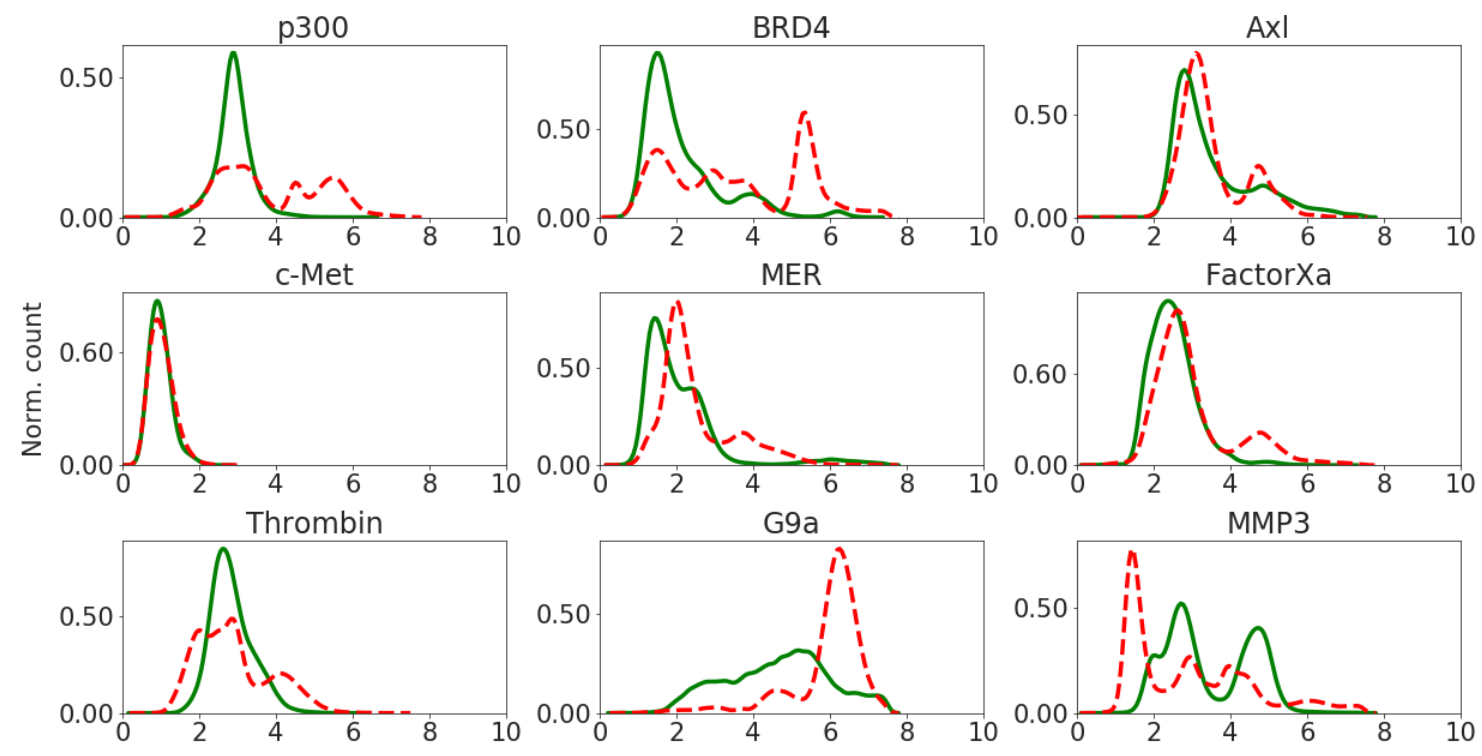

RMSD in pocket $(\AA)$

Figure S1. Probability density functions for the ligand mobility in the binding pocket expressed as RMSD with respect to the native pose in the protein coordinates (where sampled structures were aligned using all $\mathrm{C} \alpha$ atoms). 

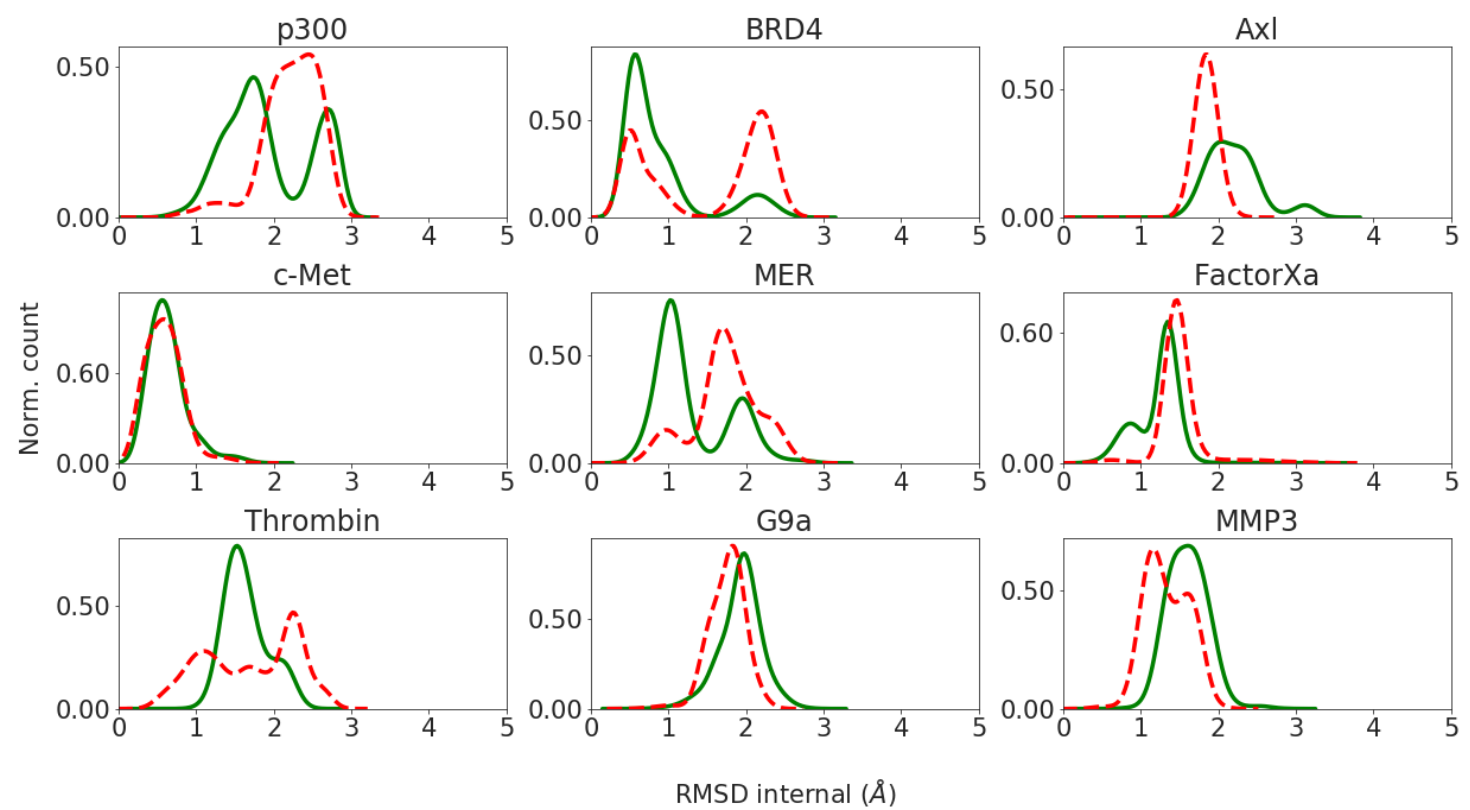

Figure S2. Probability density functions for the conformational ligand mobility expressed as $R M S D$ with respect to the native pose in the ligand coordinates (based on the ligand alignments). 

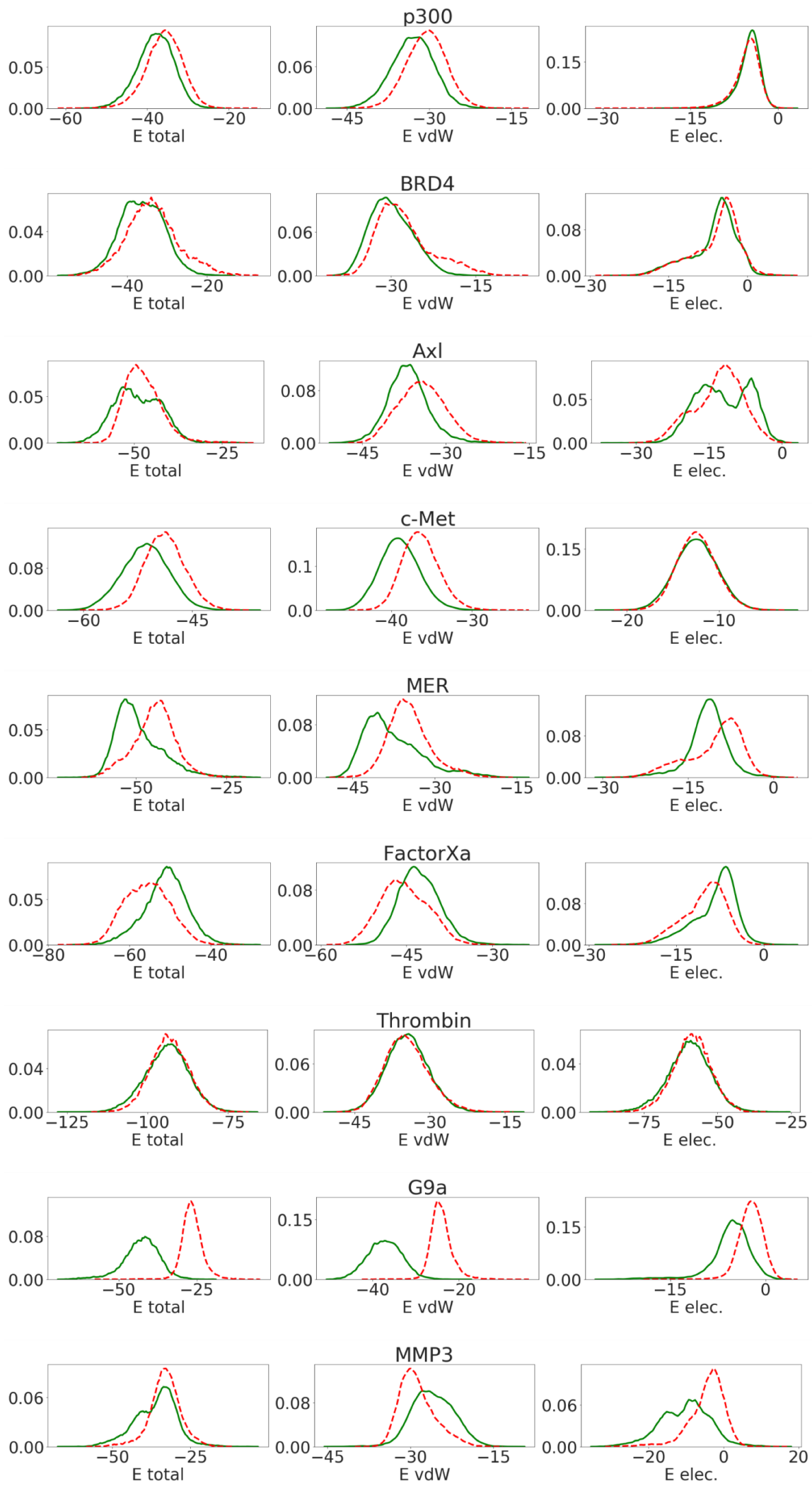

Figure S3. Probability density functions for the ligand-protein total, vdW and electrostatic interaction energies (in $\mathrm{kcal} / \mathrm{mol})$. 

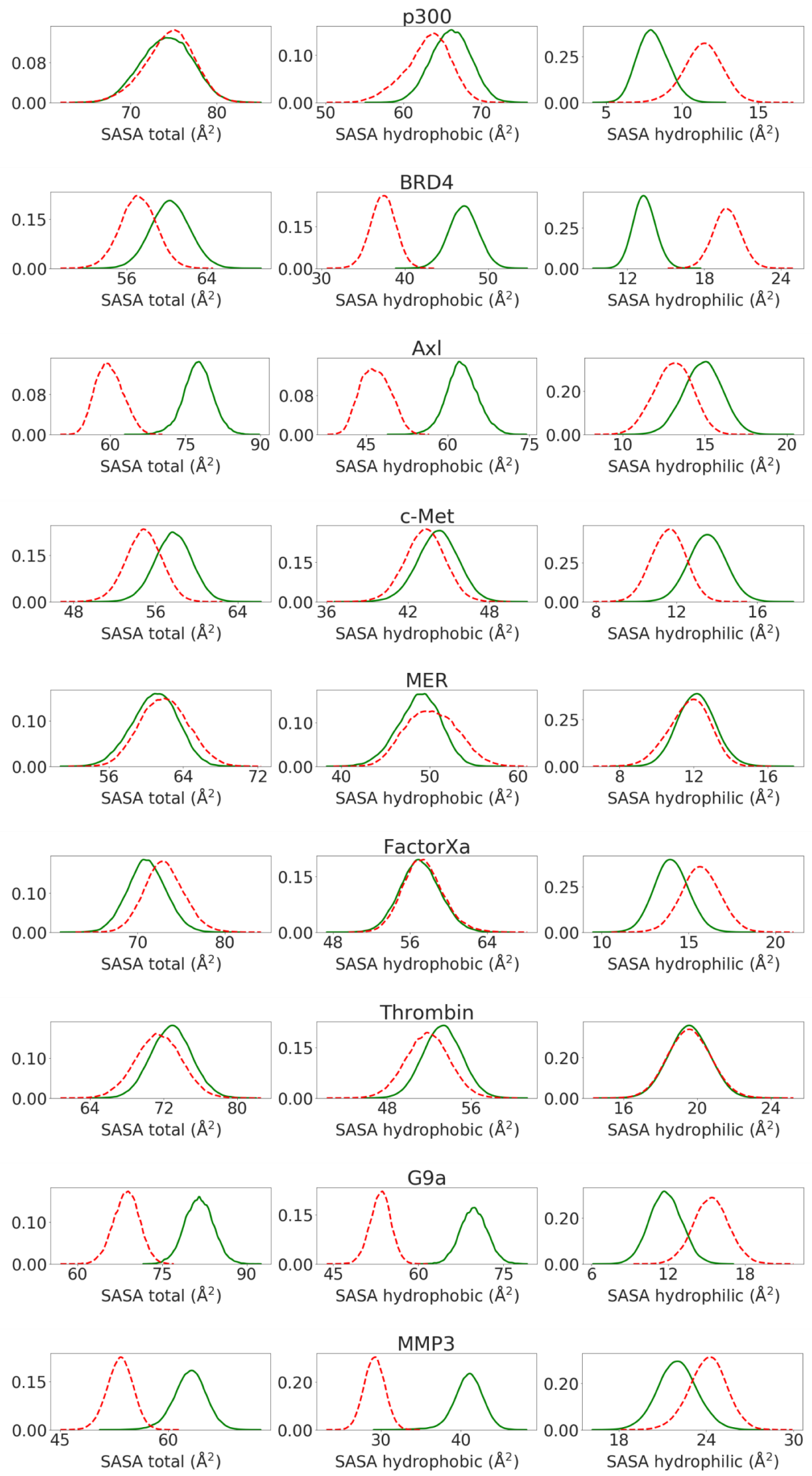

Figure S4. Probability density functions for the total, lipophilic and hydrophilic SASA of a protein-bound ligand. 

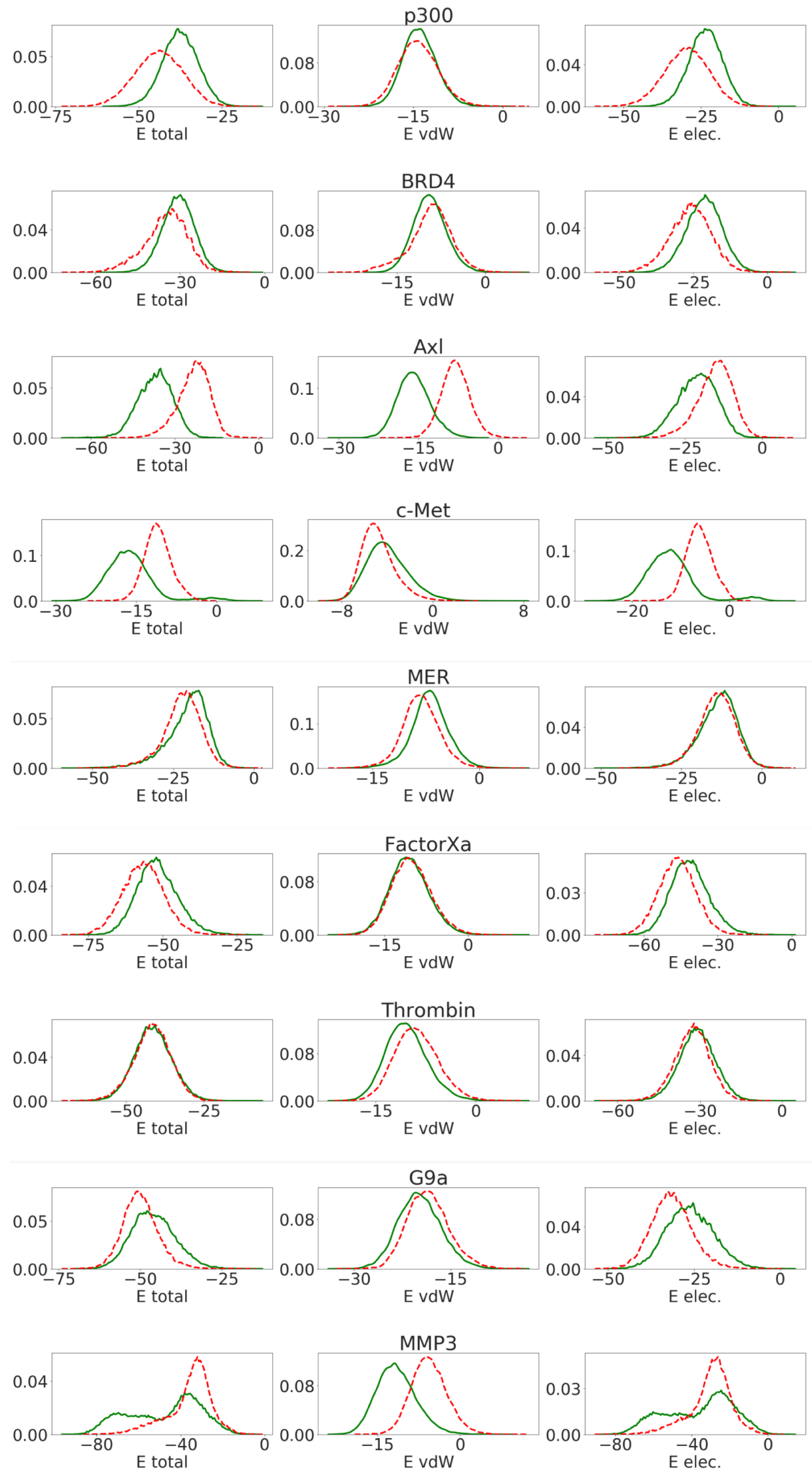

Figure S5. Probability density functions for the ligand-water total, vdW and electrostatic interaction energies (in $\mathrm{kcal} / \mathrm{mol})$. 

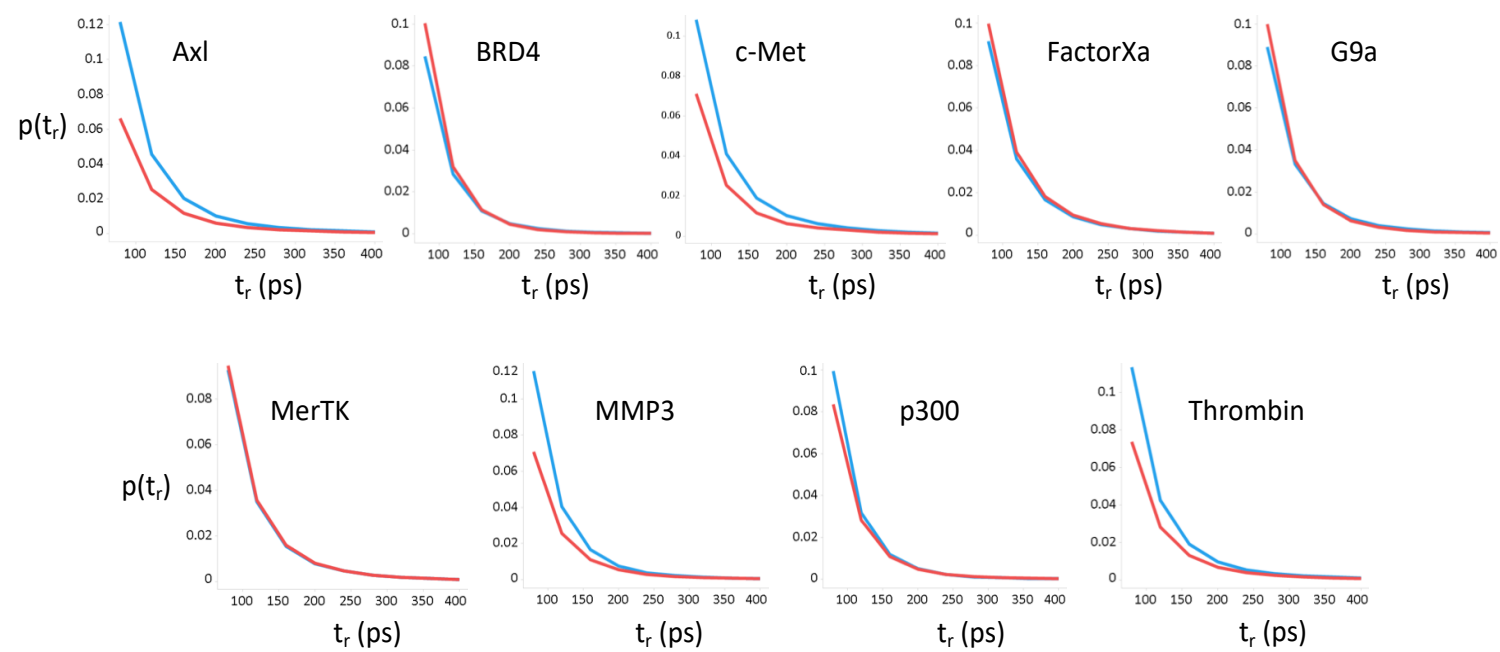

Figure S6: Probability density functions (PDF) for residence times ( $\mathrm{t}_{\mathrm{r}}$; in picoseconds) of water molecules around the ligand for all simulated protein-ligand complexes. Red and blue curves are PDFs for respectively active and inactive ligands. 


\section{0 clusters}

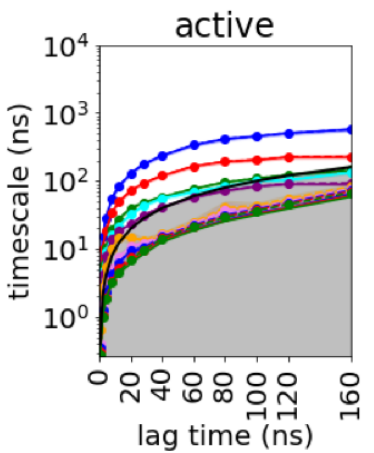

\section{0 clusters}

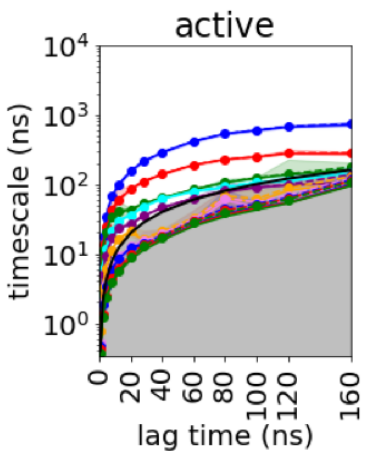

\section{0 clusters}

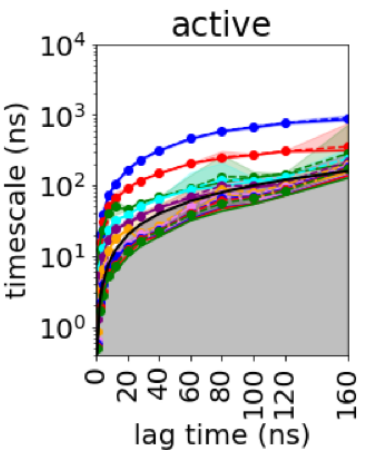

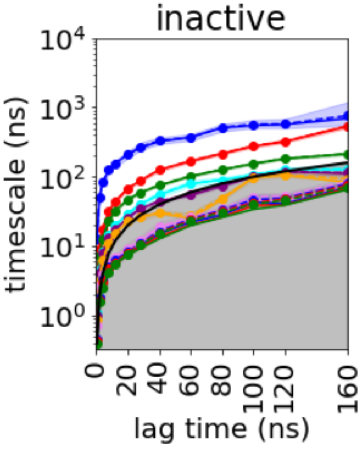
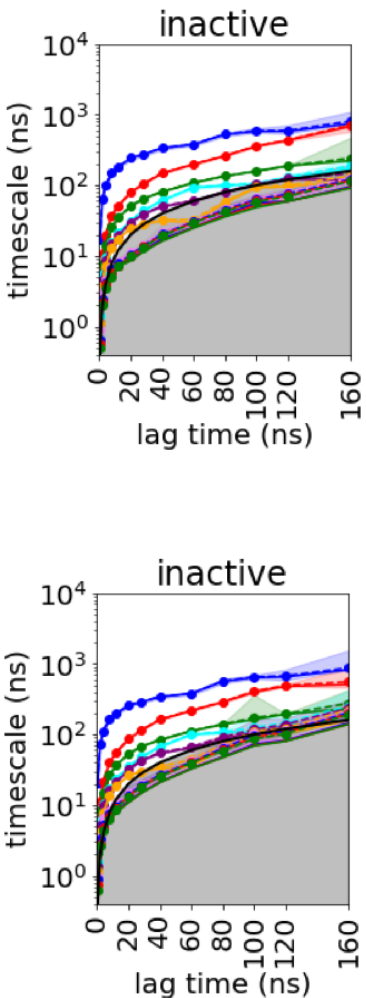

\section{0 clusters}
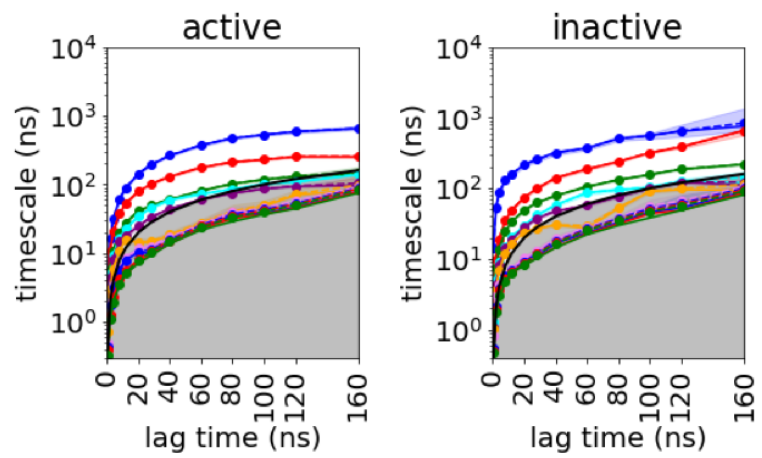

\section{0 clusters}
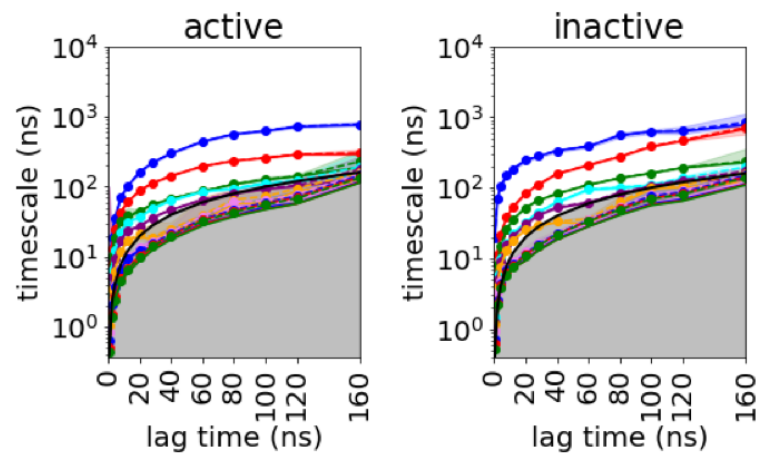

\section{0 clusters}

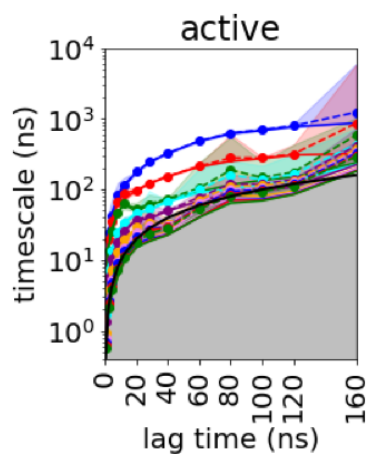

Figure S7. Implied timescales for 50, 100, 200, 300, 500, and 1000 cluster (microstate) MSMs. The graphs represent implied timescales (ITS) against lag time. In blue, red, green, ... are the 1st, 2nd, 3rd, ... slowest ITSs. The ITS of the maximum likelihood Bayesian Markov model are shown in solid lines, whereas the means and the 95\% confidence intervals (computed by Bayesian sampling) are shown in dashed and shaded areas, respectively. A lag time of 80 ns (Fig. S8) with 200 microstates for the active and 300 microstates for the inactive (Table 1) were chosen. 

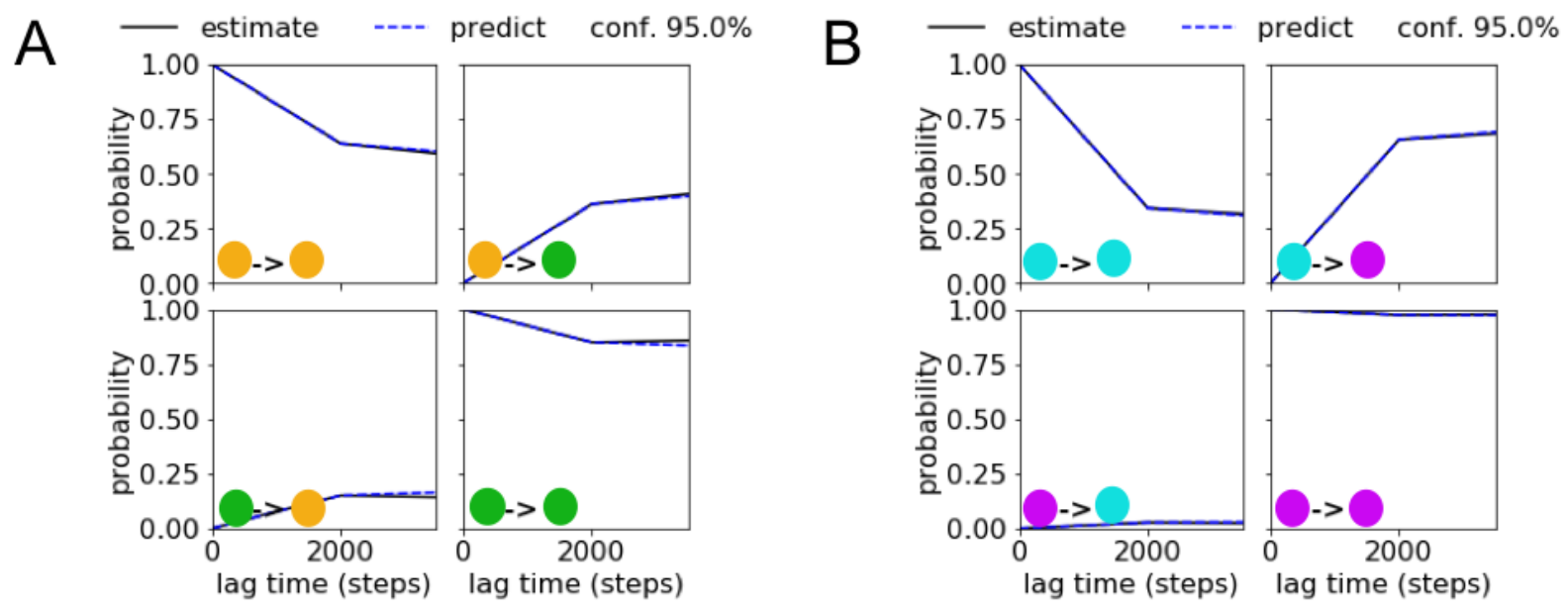

Figure S8. CK tests for MSMs of (A) active and (B) inactive systems, with 200 and 300 microstates respectively. For each of the metastable states in our models we initialize the population in that state and compare the evolution of the population predicted from the MSM at given lag times (dashed blue lines) to that measured on the trajectories (black lines) at the same lag times. The shaded areas represent $95 \%$ confidence intervals computed by bootstrapping. Additionally, we also performed $\mathrm{CH}$ tests for 20,40, and 60 ns lag times, for which MSM estimates did not correlate with the simulation prediction. Based on this result lag time of $80 \mathrm{~ns}$ was chosen (2000 steps).

\section{SUPPLEMENTARY TABLES}

Table S1. Variational scores (designated as score_cv in PyEMMA) for different clustering solutions.

\begin{tabular}{|l|l|l|}
\hline active & inactive & Number of Clusters (Microstates) \\
\hline 2.29 & 2.46 & 50 \\
\hline 2.28 & 2.48 & 100 \\
\hline 2.40 & 2.48 & 200 \\
\hline 2.28 & 2.56 & 300 \\
\hline 2.36 & 2.43 & 500 \\
\hline 2.32 & 2.33 & 1000 \\
\hline
\end{tabular}

